\title{
Tsunami-Sinusitis
}

\section{Den halben Strand in der Nase ...}

\author{
Wer von einem Tsunami mitgerissen \\ wird, kann froh sein, wenn er über- \\ lebt. Doch Meerwasser und Sand, die \\ dabei mit hoher Geschwindigkeit bis \\ in die Tiefen der Nase gelangen, be- \\ reiten noch nach Wochen Probleme - \\ es droht die Tsunami-Sinusitis.
}

Shintaro Baba und seine Kollegen aus Tokio berichten in "The Lancet" vom Fall einer Frau, die bei der Tsunami-Katastrophe an Japans Küste Anfang 2011 beinahe ertrunken war. Die 31-Jährige kam eine Woche nach dem verheerenden Erdbeben in die Uniklinik. Sie hatte die Flutwelle überlebt, aber Meerwasser aspiriert, sich die Brustwirbelsäule gebrochen und eine Lungenentzündung zugezogen.

\section{Flüssige Substanz in Kiefer- und Keillbeinhöhlen}

Die Pneumonie besserte sich unter Antibiotika, das Fieber der Frau und ihr hoher CRP-Wert sanken jedoch nicht.
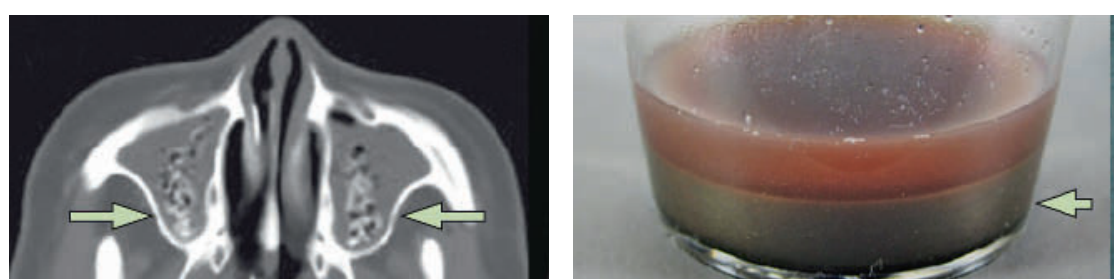

Abb. 1 Links das CT der Nebenhöhlen vor dem Spülen und rechts der sandige Inhalt.

Die Ärzte beschlossen, die Nebenhöhlen der Patientin zu spülen, da sie im CT flüssige Substanz in Kiefer- und Keilbeinhöhlen entdeckt hatten (• Abb. 1).

\section{Mix aus Bakterien}

Unter lokaler Anästhesie förderten sie grünes, eitriges Sekret und Sand aus beiden Kieferhöhlen. Bereits am nächsten Tag ging ihr Fieber zurück und der CRPWert sank. Drei Wochen später klagte die Frau über starke Kopfschmerzen. Ihr CRP-Wert war wieder gestiegen und noch immer füllte flüssige Substanz die Keilbeinhöhlen. Erst als die Ärzte auch dort Eiter und Sand entfernten, besserte sich der Zustand der Patientin dauerhaft.
Die Entzündung ausgelöst hatten Bakterien, die vermutlich mit verunreinigtem Meerwasser in die Nebenhöhlen gelangt waren. Die Ärzte konnten Pseudomonas aeruginosa, Proteus vulgaris und Escherichia coli nachweisen.

Bereits bei der Tsunami-Katastrophe in Thailand 2004 hatten Ärzte einen ganz ähnlichen Fall unter dem Titel „Tsunami-Sinusitis“ publiziert. Auch in diesem Fall waren Sand und Eiter in den Nebenhöhlen. Eine einheitliche Behandlung der Tsunami-Sinusitis hat sich bisher aber nicht etabliert. Die charakteristische Sinusitis zu kennen, empfehlen die Ärzte aus Tokio jedoch jedem, der Tsunami-Opfer behandelt. $\rightarrow$ einem Rachenabstrich für einen GASSchnelltest geraten. Ein mehrtägiger Kulturnachweis ist dagegen für die Therapieentscheidung nicht ratsam.

\section{Sinnvolle symptomatische Therapie}

Häufig wird der generelle Wunsch des Patienten nach Antibiotika überschätzt oder ist sogar missverständlich, denn es steckt oft nur die schlichte Hoffnung auf Schmerzlinderung dahinter. Diese erfolgt am besten mit Paracetamol und Ibuprofen. Auch bei Antibiotikabehandlung reduzieren regelmäßig eingenommene Analgetika die Symptome in den ersten Tagen. Empfehlen kann man auch einfache Maßnahmen, wie vermehrtes
Trinken, Gurgeln mit Salzwasser oder Tee, Lutschen nichtmedizinischer Bonbons oder Halswickel.

Lokalantiseptika und -anästhetika, ob in Form von Lutschtabletten, Gurgellösung oder als Rachenspray, wirken dagegen nur oberflächig, erreichen damit nicht die tiefer sitzende wesentliche Infektion und sind deshalb auch nicht sinnvoll.

(Melanie Goldschmidt) HNO 2011, 59:480

\section{Abb. Algorithmus zur Therapieentscheidung.}

Wird die Wirkung von Antibiotika als relevant eingeschätzt? (abhängig vom Schweregrad der Erkrankung)

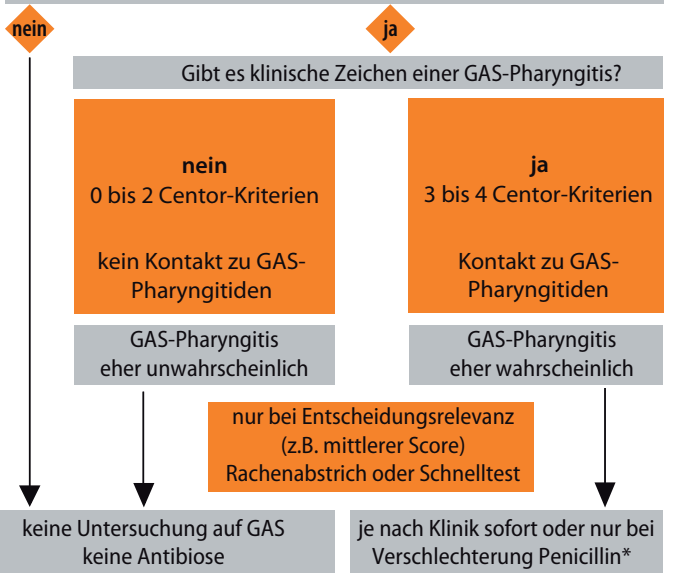

* bei Unverträglichkeit Erythromycin 